\title{
Magnetic resonance imaging biomarkers in hepatocellular carcinoma: association with response and circulating biomarkers after sunitinib therapy
}

Dushyant V Sahani ${ }^{1 *}$, Tao Jiang ${ }^{1,2}$, Koichi Hayano ${ }^{1}$, Dan G Duda ${ }^{3}$, Onofrio A Catalano ${ }^{1,4}$, Marek Ancukiewicz ${ }^{3}$, Rakesh K Jain ${ }^{3}$ and Andrew X Zhu ${ }^{5}$

\begin{abstract}
Background: To investigate the hypothesis that MRI derived diffusion-weighted imaging (DWI) and perfusion (MRP) parameters are sensitive image biomarkers for monitoring early antiangiogenic effects and predicting progression free survival (PFS) in advanced hepatocellular carcinoma (HCC).

Methods: In this phase II clinical trial, 23 of 34 patients were included in the imaging and circulating biomarker study. DWI and MRP were performed at the baseline and at 2-weeks after initiation of sunitinib. The imaging protocol included an axial DWI sequence using b values of 50,400 and $800 \mathrm{sec} / \mathrm{mm}^{2}$, and MRP using a series of coronal 3D-VIBE following $20 \mathrm{ml}$ of Gd-DTPA at $2 \mathrm{ml} / \mathrm{sec}$. These parameters were compared with clinical outcome and PFS at 6-months. Correlation between changes in MRI parameters and plasma biomarkers was also evaluated.

Results: After 2-week of sunitinib, substantial Ktrans changes in HCC were observed from median baseline value $2.15 \mathrm{~min}^{-1}$ to $0.94 \mathrm{~min}^{-1}(P=0.0001)$ with increases in median apparent diffusion coefficient (ADC) from $0.88 \times 10^{-3}$ $\mathrm{mm}^{2} / \mathrm{s}$ to $0.98 \times 10^{-3} \mathrm{~mm}^{2} / \mathrm{s}(P=0.0001)$. Tumor size remained unchanged by RECIST and mRECIST (both $\left.P>0.05\right)$. Patients who showed larger drop in Ktrans and Kep at 2 weeks correlated with favorable clinical outcome, and higher baseline Ktrans and larger drop in EVF correlated with longer PFS (all $P<0.05$ ). There was a significant association between a decrease in SVEGFR2 and the drop in Ktrans and Kep $(P=0.044, P=0.030)$, and a significant and borderline association between decrease in TNF- $a$ and the drop in Ktrans and Kep, respectively $(P=0.051, P=0.035)$.
\end{abstract}

Conclusion: In HCC, MRP may be a more sensitive biomarker in predicting early response and PFS following sunitinib than RECIST and mRECIST.

Trial registration: ClinicalTrials.gov: NCT00361309

Keywords: Hepatocellular carcinoma, Antiangiogenic treatment, Image biomarker, Dynamic contrast-enhanced MRI, Diffusion-weighted imaging, Circulating biomarker

\footnotetext{
* Correspondence: dsahani@partners.org

'Division of Abdominal Imaging and Intervention, Harvard Medical School and Massachusetts General Hospital, 55 Fruit Street, White 270, Boston, MA 02114, USA

Full list of author information is available at the end of the article
} 


\section{Background}

Hepatocellular carcinoma (HCC) is the sixth most common cancer and the third most common cause of cancerrelated death worldwide and is responsible for more than 500,000 deaths every year globally [1]. Advanced HCC carries a poor prognosis, and systemic therapy with cytotoxic agents provides marginal benefit [2,3]. Over the past few years, significant progress has been made in our understanding of the molecular pathogenesis of HCC, which led to the rationale for the use of novel targeted agents in clinical trials. Due to highly angiogenic microenvironment in HCC, antiangiogenic agents such as sorafenib, bevacizumab and cediranib have been tested in clinical trials for the treatment of HCC [4-10]. While sorafenib has shown increased survival in HCC, other anti-VEGF agents have shown mixed results [11]. Sunitinib (Sutent; Pfizer, New York, NY) is an oral multitargeted receptor tyrosine kinase inhibitor (TKI), which has been approved for the treatment of renal cell carcinoma, imatinib-resistant gastrointestinal stromal and pancreatic neuroendocrine tumors [12]. Although sunitinib demonstrated early evidence of modest antitumor activity in advanced HCC patients from single arm phase II studies [13], a randomized phase III trial failed to demonstrate either superiority or non-inferiority of sunitinib when compared with sorafenib. This clinical experience indicates that only some patients benefit from these targeted therapies. The mechanisms of action in targeted agents, which often cause cytostatic effect rather than cytotoxic effect, are different from conventional chemotherapy, and therefore, the response assessment criteria currently in use might be inadequate. Imaging modalities such as computed tomography (CT) and magnetic resonance imaging (MRI) are commonly used in phases II and III clinical trials. They provide reliable and reproducible anatomical assessment of changes in tumor size. However, antiangiogenic therapies for HCC are known to induce tumor necrosis and may cause nochange or relative enlargement of the tumor size on imaging thereby leading to inappropriate categorization of an otherwise responsive disease, as stable or progressive based on established methods such as Response Evaluation Criteria In Solid Tumors (RECIST) [14]. Therefore, the European Association for the Study of the Liver (EASL) guidelines recommended that the response criteria be amended to take into account viable tumor (contrast enhancement in the arterial phase) [15], and the American Association for the Study of Liver Disease (AASLD) developed a set of guidelines that included a formal modification of the response assessment based on the RECIST criteria and aimed to translate into the concept of viable tumor, which are referred to as modified RECIST (mRECIST) criteria [16].

With the advancements in MR technology and availability of commercial software, MR perfusion (MRP) and diffusion-weighted imaging (DWI) have found their applications in HCC [17]. DWI uses phase-defocusing and phase-refocusing gradients, which allow evaluation of the rate of microscopic water diffusion as a marker of cellular density and integrity. Using a dynamic MRI, hemodynamic parameters for permeability measurement, such as transfer constant (Ktrans), redistribution rate constant (Kep) and extracellular volume fraction (EVF), can be quantitated [18]. Several studies had attempted to assess if DWI and MRP derived tumor parameters could be used for assessment of response to therapies [12,19-21]. Therefore, we had formulated and investigated the hypothesis that the DWI or MRP derived tumor parameters are more sensitive image biomarkers when compared with tumor burden measurements as defined by RECIST or mRECIST in a clinical trial of sunitinib for monitoring early antiangiogenic effects and predicting progression free survival (PFS) in advanced HCC. We also postulated that imaging biomarkers correlate with circulating biomarkers measured in plasma. Additional objective was to compare the DWI and MRP parameters of tumor thrombus and their changes in patients with different clinical outcome and PFS.

\section{Methods}

\section{Patients}

The protocol for this phase II clinical trial was in compliance with Health Insurance Portability and Accountability Act (HIPAA) Regulations and was approved by the The Institutional Review Board (IRB) at Dana Farber Harvard Cancer Center. All patients were required to provide written informed consent before study participation according to institutional and federal guidelines. Eligibility criteria included histologically proven, measurable, locally advanced, recurrent or metastatic $\mathrm{HCC}$; no more than one prior systemic regimen; prior chemoembolization therapy only if performed more than 4 weeks before study entry and measurable disease present outside of the chemoembolization field; age 18 years; Eastern Cooperative Oncology Group performance status of 0 or 1; Cancer of the Liver Italian Program (CLIP) score 0-3; and adequate hepatic, renal, and bone marrow function. Exclusion criteria included concurrent malignancies; significant medical comorbidities; significant cardiovascular disease including uncontrolled hypertension, myocardial infarction, and unstable angina; New York Heart Association grade 2 or greater congestive heart failure; prolongation of QTc of more than $450 \mathrm{msec}$ in screening ECG or history of familial long QT syndrome; history of bleeding; proteinuria at baseline (more than $2 \mathrm{~g} / \mathrm{d}$ ); pregnancy or lactation; central nervous system metastases; or an inability to provide written informed consent. Thirtyfour patients with advanced HCC were enrolled and 23 
were included in the current study. The prospective study cohort included 7 men and 16 women (age range, $38 \sim 76$ years; median age, 62.6 years).

\section{Antiangiogenic treatment}

The eligibility, treatment schedule, and dose modification schema have been detailed previously [10]. Briefly, eligible patients received sunitinib at a dose of $37.5 \mathrm{mg}$ daily by mouth for 28 days followed by 14 days of rest in 6-week cycles. Patients with grade 3 or 4 toxicities underwent dose reduction to 25 or $12.5 \mathrm{mg}$ daily, respectively. Treatment was continued until progression, unacceptable toxicity, or withdrawal of consent. Response and progression were evaluated using the RECIST after completion two cycles of sunitinib therapy.

\section{Imaging protocol}

This clinical trial was designed not only to investigate the role of DWI and MRP for monitoring early antiangiogenic treatment effects but also to study the overall survival and PFS. The DWI, MRP and delayed postcontrast T1weighted images were performed at base line and two weeks after initiation of antiangiogenic treatment. A restaging contrast-enhanced MRI (CE-MRI) was performed at the end of cycle 2 treatment with sunitinib for response status and then at every 6-weeks until disease progression. The data acquisition parameters, the same injection protocol and the anatomic location for scanning, including the total duration, were kept constant for each patient and for each repeat DWI, MRP and CE-MRI study.

\section{DWI}

DWI of the liver was performed using a phased array body coil on a 1.5-T MRI system (Avento; Siemens, New York, NY) using the following protocol. T1-weighted in-phase and out- of-phase images (repetition time (TR)/ echo time (TE), 122 159/2.38 $4.72 \mathrm{msec}$; one signal acquired; flip angle, 70 degree; 20 slices; section thickness, $5 \sim 7 \mathrm{~mm}$; 1-mm interslice gap) and axial respiratory-triggered fast spin-echo T2-weighted images (TR/TE, $3500 \sim 4494 / 65 \sim 85 \mathrm{msec}$; one signal acquired; flip angle, 53 degree; echo train length, 15; 20 slices; section thickness, $5 \sim 8 \mathrm{~mm}$; 1-mm interslice gap) were acquired first. Thereafter, an axial respiratory-gated echo-planar diffusion-weighted (DW) sequence with spectral fat saturation was performed by using the following parameters: b values of 50,400 and $800 \mathrm{sec} / \mathrm{mm}^{2}$; TR/TE, 4959 7936/44 74 msec; two signals acquired; echo train length, 1; flip angle, $60 \sim 90$ degree; 20 slices; section thickness, $5 \sim 8 \mathrm{~mm}$; 1-mm interslice gap; field of view, $263 \times 350$; matrix, $144 \times 192$. The total acquisition time is $3 \sim 6 \mathrm{~min}$.

\section{MRP}

MRP of the liver was performed using a phased array body coil on the same 1.5-T MRI system (Avento; Siemens, New York, NY) using the following protocol. At first, three dimensional volume interpolated excitation coronal T1 sequence (VIBE) was obtained in a breath hold before contrast media injection using the following parameters: $\mathrm{TR}=5 \mathrm{msec}, \mathrm{TE}=1.58 \mathrm{msec}, 5-\mathrm{mm}$ slice thickness, $1-\mathrm{mm}$ interslice gap, 20 slices, $123 \times 192$ matrix, and field of view of $400 \times 400 \mathrm{~mm}$. Thereafter, through the 20 -guage peripheral intravenous line in the arm, $0.1 \mathrm{mmol} / \mathrm{kg}$ body weight of gadopentetate dimeglumide contrast media (Magnevist; Berlex, Montville, NJ) was power injected at $2 \mathrm{~mL} / \mathrm{sec}$, followed by a saline chase of $20 \mathrm{~mL}$ at a rate of $2 \mathrm{~mL} / \mathrm{sec}$. Then, MRP acquisition was performed. A series of coronal T1-weighted three-dimensional volume interpolated excitation (VIBE) images were obtained after 5-second delay after the initiation of contrast media injection, and the scanning continued for up to 4 minutes and $40 \mathrm{sec}-$ onds. The acquisition parameters included: $\mathrm{TR}=5 \mathrm{msec}$, $\mathrm{TE}=1.58 \mathrm{msec}$, 5-mm slice thickness, 0 - $\mathrm{mm}$ interslice gap, 20 slices, $123 \times 192$ matrix, 15-degree flip angle, and field of view of $400 \times 400 \mathrm{~mm}$. Two consecutive 7-second acquisitions forming two different time points were repeated 10 times with a delay of 14 seconds between them. The scanning time in every acquisition was 14 seconds with a break of 14 seconds, and the patients were asked to hold their breath during acquisition. Finally, delayed postcontrast T1weighted images were taken as follows: axial and coronal two-dimensional T1-weighted fat-saturated gradient echo (GRE) sequences using TR $=150 \mathrm{msec}, \mathrm{TE}=2.1 \mathrm{msec}$, $160 \times 256$ matrix, 20 slices, 5-mm thickness, and $0-\mathrm{mm}$ interslice gap. For the measurement of tumor burden and diameter of tumor thrombus in portal vein, postcontrast T1-weighted images were applied.

\section{CE-MRI}

CE-MRI of the liver was performed using a phased array body coil on the same 1.5-T MRI system (Avento; Siemens, New York, NY) using the following protocol. A total of $0.1 \mathrm{mmol} / \mathrm{kg}$ body weight of gadoliniumdiethylenetriaminepentaacetic acid (Gd-DTPA) contrast media (Magnevist; Berlex, Montville, NJ) was power injected at $2 \mathrm{~mL} / \mathrm{sec}$, followed by a saline chase of $20 \mathrm{~mL}$ at a rate of $2 \mathrm{~mL} / \mathrm{sec}$. The arterial, portal and delayed phase scanning were performed after 35, 65 and 280 seconds from the initiation of contrast medium bolus. Three dimensional volume interpolated excitation axial T1 sequence was obtained in a breath hold after contrast media injection using the following parameters: $\mathrm{TR}=5 \mathrm{msec}, \mathrm{TE}=1.58 \mathrm{msec}, 5-\mathrm{mm}$ slice thickness, $0-\mathrm{mm}$ interslice gap, 20 slices, $123 \times 192$ matrix, and field of view of $400 \times 400 \mathrm{~mm}$. 


\section{Image processing}

Data were processed at a picture archiving and communication system (PACS) (Impax 4.0; Agfa, Mortsel, Belgium) by two experienced radiologists with 13 and 10 years of experience in liver imaging. To obtain permeability maps, MRP images were processed at pixel resolution by using a commercially available full time point (fTP) model (iCAD Sciences, White Plains, NY) to analyze the time evolution of contrast enhancement.

Tumor size was measured in the longest cross-sectional dimension for each lesion based on RECIST 1.1 guidelines. And viable tumor diameter was measured in the longest cross-sectional dimension for enhancing component of each lesion based on mRECIST guidelines. The sum of the longest dimensions of selected target lesions in each patient was computed, and the absolute and percent changes of the sum from the baseline to post treatment evaluation were computed for each patient. Diagnostic standard and diameter measurement for tumor thrombus in portal vein was referred to the criteria described by Shah et al. [22].

The apparent diffusion coefficient (ADC) map was automatically generated on the imager console from the DWI sequence; the selected b values (50, 400 and $800 \mathrm{sec} / \mathrm{mm}^{2}$ ) were used for $\mathrm{ADC}$ quantification. For $\mathrm{ADC}$ quantitative analysis, the nonenhanced images showing the maximal diameter of the $\mathrm{HCC}$ and the maximal diameter of the tumor thrombus in the portal vein were respectively selected as reference [23]. Definitions of MRP parameters and the model used for generating functional maps were described as before [24,25]. Briefly, for each MRP acquisition, the fTP-pharmacokinetic image analysis platform implements the Tofts pharmacokinetic model to quantify vascular permeability (Ktrans, EVF and Kep).

On DWI and MRP postprocessed images, we manually drew region of interests (ROI) in all the anatomic locations from the section in which the tumor was first visible to the last section in which the tumor was visible to enable whole tumor evaluation, and corresponding values were acquired. ROI included at least two-thirds of the area of the HCC and at least three-fourths of the area of the thrombus in the portal vein. To minimize volume averaging, we enlarged the images and placed the ROI within the thrombus. Care was taken to avoid including in the ROI any area outside the HCC and the thrombus. For patients with multiple lesions, we drew ROI for all and computed a median value for analysis of parameters. Then, the absolute and percent changes in ADC value and MRP parameters from the baseline and after therapy were calculated for each patient.

\section{Circulating biomarkers}

For measurement of angiogenic proteins and inflammatory cytokines in plasma, peripheral blood was obtained at baseline and 14 days after the first dose of sunitinib.
All samples were collected in ethylenediaminetetraacetic acid (EDTA)-containing Vacutainers. Plasma analysis was carried out for circulating vascular endothelial growth factor (VEGF), placental-derived growth factor (PIGF), soluble VEGF receptor (VEGFR) 1, basic fibroblast growth factor (bFGF), interleukin (IL)-1 $\beta$, IL-6, IL-8, and tumor necrosis factor $\alpha$ (TNF- $\alpha)$ using multiplex array plates from Meso-Scale Discovery (Gaithersburg, MD), and for soluble VEGFR2, soluble VEGFR3, stromalderived factor $1 \alpha(\mathrm{SDF} 1 \alpha)$, VEGF-C, and soluble c-KIT from R\&D Systems (Minneapolis, MN) [12]. Samples were run in duplicate.

\section{Statistical analysis}

Data were analyzed using SPSS statistical software, version 10.0, SPSS Inc, Chicago, IL and R ( R Foundation for Statistical Computing, Vienna, Austria). We present medians with ranges of various parameters of the HCC and tumor thrombus at baseline and following antiangiogenic treatment. Changes in ADC value and MRP parameters of HCC and tumor thrombus as well as tumor burden measures were expressed as percent changes and tested statistically using paired exact Wilcoxon signed rank test for comparison at the baseline and at the 2 weeks after initiation of treatment. For correlation with clinical outcome, patients were divided into two groups: those with progressive disease (PD) and those with either stable disease (SD) or a partial response (PR). Similarly, for correlation with PFS, patients were divided into two groups: those with PFS $\leq 6$ months and those with PFS $>6$ months. The baseline values and percent changes in various parameters after sunitinib administration in these groups were compared using the two-sample exact Wilcoxon rank sum test. Correlation between changes in MRI parameters and plasma biomarkers at day 15 after sunitinib treatment in advanced HCC patients was performed using Kendall's correlation coefficients. In this study, a $P$-value of less than or equal to 0.05 was considered to indicate a statistically significant difference.

\section{Results}

The clinical outcome of patients from the phase II trial has been previously reported [10]. Briefly, of the 23 patients enrolled in the current study who were evaluable for efficacy after completion two cycles of sunitinib therapy, one (4.4\%) had a confirmed PR and 15 (65.2\%) had SD, the other $7(30.4 \%)$ showed PD. In 14 patients (60.9\%) PFS $\leq 6$ months was encountered and the other 9 had PFS $>6$ months (39.1\%). In addition, 9 of 23 patients $(39.1 \%)$ were categorized as having tumor thrombosis; 5 (55.6\%) showed PFS $\leq 6$ months and the other 4 had PFS $>6$ months (44.4\%). 
Table 1 Tumor and tumor thrombus size, DWI and MRP parameters at baseline and post antiangiogenic treatment

\begin{tabular}{|c|c|c|c|c|}
\hline \multicolumn{2}{|l|}{ Parameters } & Baseline (Median, Range) & Post-sunitinib (Median, Range) & $P$ value \\
\hline \multicolumn{5}{|l|}{ SIZE } \\
\hline \multicolumn{2}{|l|}{ RECIST } & $10.00,2.30 \sim 17.00$ & $11.10,1.30 \sim 25.00$ & 0.197 \\
\hline \multicolumn{2}{|l|}{ m RECIST } & $9.94,2.15 \sim 19.56$ & $10.11,2.11 \sim 18.55$ & 0.963 \\
\hline \multicolumn{2}{|c|}{ Tumor thrombus(mm) } & $28.67,21.87 \sim 36.63$ & $29.81,21.98 \sim 37.73$ & 0.365 \\
\hline \multicolumn{5}{|l|}{ DWI } \\
\hline $\mathrm{HCC}$ & $A D C\left(\times 10^{-3} \mathrm{~mm}^{2} / \mathrm{s}\right)$ & $0.88,0.58 \sim 1.09$ & $0.98,0.75 \sim 1.21$ & $<0.0001$ \\
\hline Tumor thrombus & $A D C\left(\times 10^{-3} \mathrm{~mm}^{2} / \mathrm{s}\right)$ & $0.89,0.60 \sim 1.14$ & $1.02,0.78 \sim 1.26$ & 0.035 \\
\hline \multicolumn{5}{|l|}{ MRP } \\
\hline \multirow[t]{3}{*}{$\mathrm{HCC}$} & Ktrans $\left(\min ^{-1}\right)$ & $2.15,0.90 \sim 4.81$ & $0.94,0.57 \sim 2.30$ & $<0.0001$ \\
\hline & $\operatorname{Kep}\left(\min ^{-1}\right)$ & $2.61,1.00 \sim 41.75$ & $1.22,0.66 \sim 2.64$ & $<0.0001$ \\
\hline & EVF & $0.926,0.34 \sim 1.00$ & $0.81,0.65 \sim 0.97$ & 0.079 \\
\hline \multirow[t]{3}{*}{ Tumor thrombus } & Ktrans $\left(\min ^{-1}\right)$ & $1.89,0.93 \sim 2.99$ & $0.99,0.37 \sim 1.61$ & 0.110 \\
\hline & Kep $\left(\min ^{-1}\right)$ & $2.37,1.05 \sim 4.03$ & $1.49,0.61 \sim 2.37$ & 0.190 \\
\hline & EVF & $0.83,0.70 \sim 0.98$ & $0.66,0.56 \sim 0.77$ & 0.055 \\
\hline
\end{tabular}

Abbreviations: Ktrans Transfer constant; Kep Rate constant; EVF Extracellular volume fraction; ADC Apparent diffusion coefficient.

RECIST, mRECIST, DWI and MRP parameters at baseline and after 2-week of sunitinib therapy in HCC and tumor thrombus

The tumor parameters at baseline and 2-week postsunitinib therapy are shown in Table 1. There was minimal change in median tumor burden based on RECIST (from $10.00 \mathrm{~cm}$ to $11.10 \mathrm{~cm}, P=0.197$ ) and mRECIST (from $9.94 \mathrm{~cm}$ to $10.11 \mathrm{~cm}, P=0.96$ ) and median tumor thrombus diameter (from $28.67 \mathrm{~mm}$ to $29.81 \mathrm{~mm}, P=0.365)$. There was an increase in

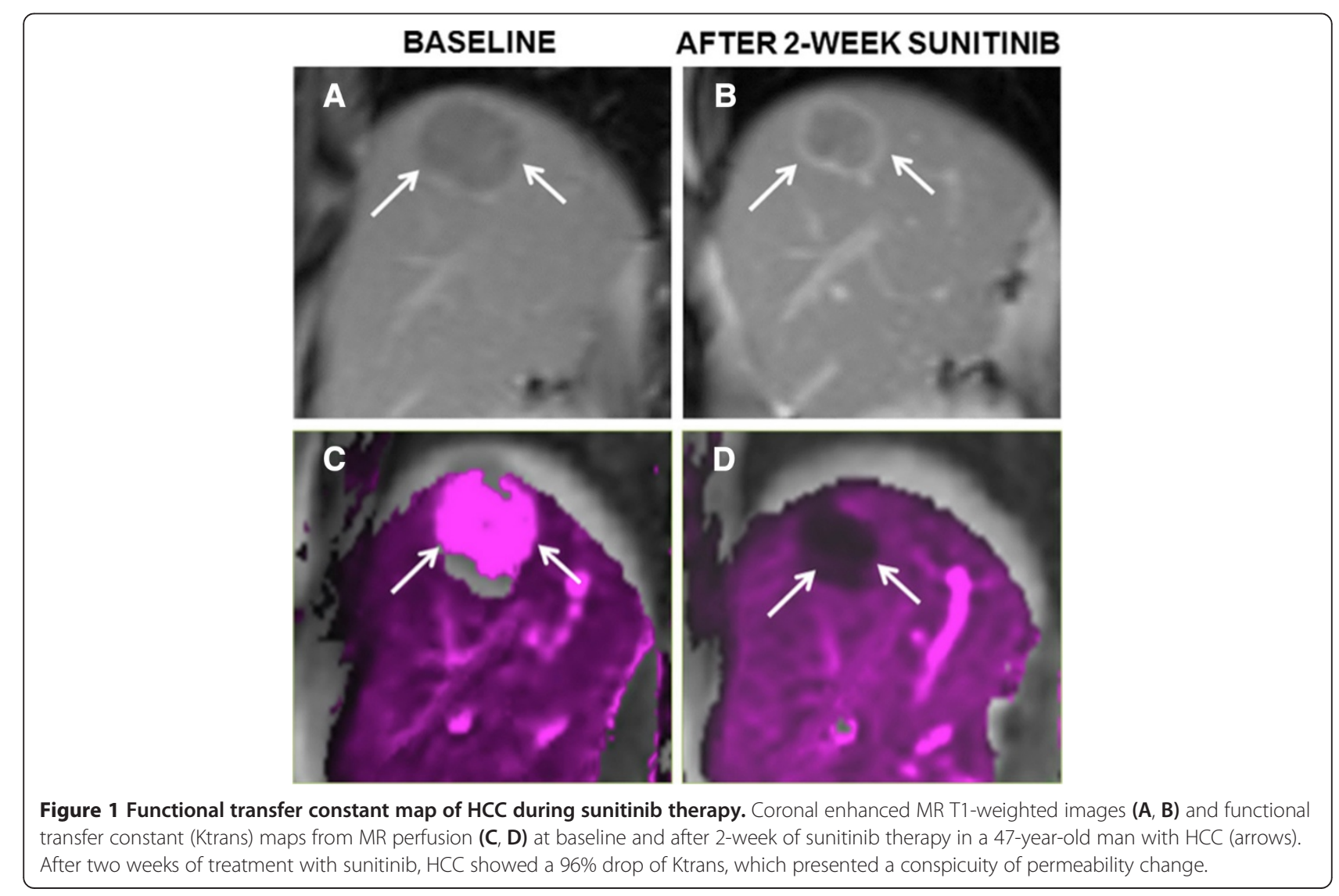



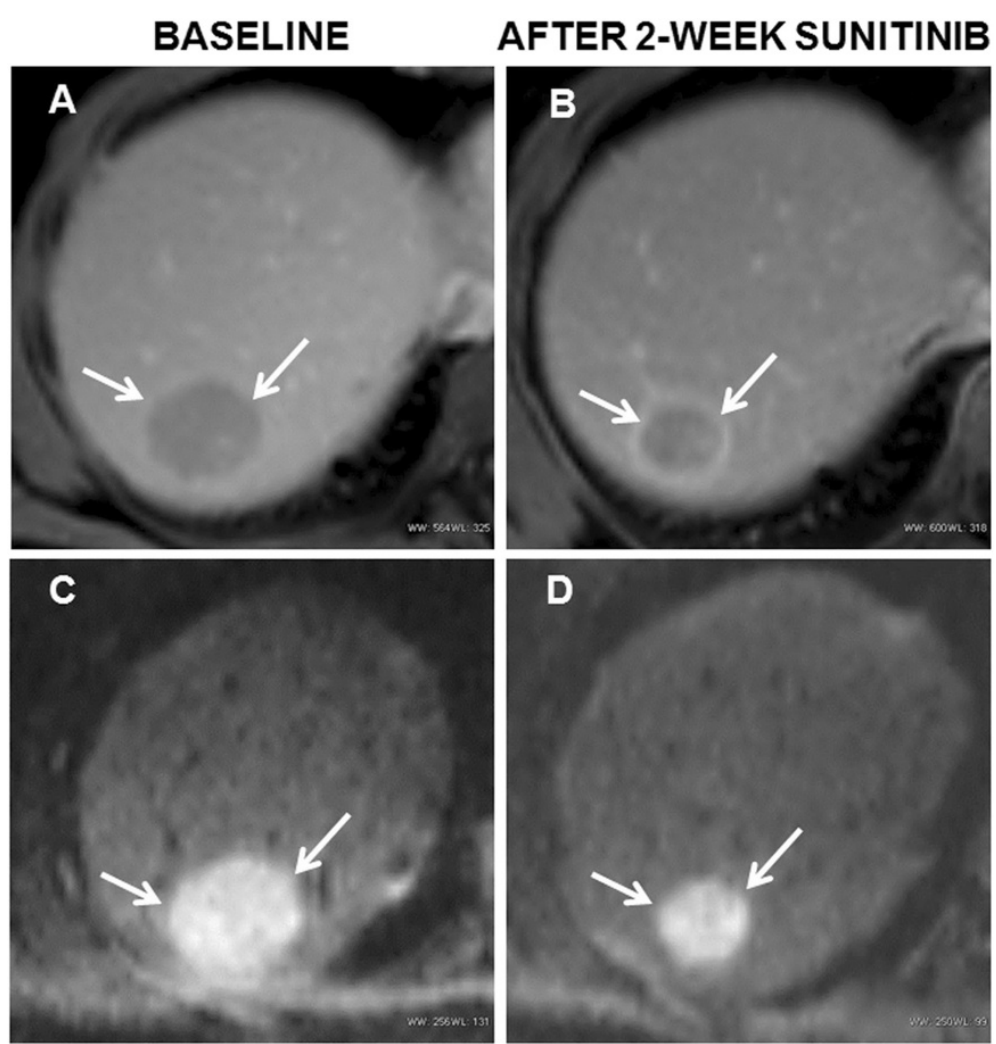

Figure 2 MR diffusion weighted images during sunitinib therapy. Transverse enhanced MR T1-weighted images (A, B) and MR diffusion weighted images (DWIs) for estimated apparent diffusion coefficient (ADC) $(\mathbf{C}, \mathbf{D})$ at baseline and after 2-week of sunitinib therapy in the same 47-year-old man with HCC (arrows). After two weeks of treatment with sunitinib, there was an increase in ADC value from $0.86 \times 10-3 \mathrm{~mm} 2 / \mathrm{s}$ to $0.94 \times 10-3 \mathrm{~mm} 2 / \mathrm{s}$.

median tumor ADC value from $0.88 \times 10^{-3} \mathrm{~mm}^{2} / \mathrm{s}$ to $0.98 \times 10^{-3} \mathrm{~mm}^{2} / \mathrm{s} \quad(P<0.0001)$ and in median tumor thrombus ADC value from $0.89 \times 10^{-3} \mathrm{~mm}^{2} / \mathrm{s}$ to $1.02 \times 10^{-}$ ${ }^{3} \mathrm{~mm}^{2} / \mathrm{s}(P=0.035)$. The Ktrans change of HCC in MRP was more substantial with a decrease from median baseline value of $2.15 \mathrm{~min}^{-1}$ to 2 -week post-treatment value of $0.94 \mathrm{~min}^{-1}(P<0.0001)$. The HCC also showed relatively higher median Kep at baseline $\left(4.15 \mathrm{~min}^{-1}\right)$ and a significant decrease compared with posttreatment value $\left(1.44 \mathrm{~min}^{-1}\right)(P<0.0001)$. The tumor thrombus displayed similar diffusion and perfusion features as their primary HCC at baseline and after treatment. The examples of Ktrans and ADC changes in an indexed HCC lesion after sunitinib administration are illustrated in Figures 1 and 2.

\section{Correlation of baseline and percent changes in DWI and MRP parameters with clinical outcome}

For correlation with clinical outcome, patients were divided into two groups: responders (RR) and nonresponders (NR) after completion of 2-cycles (12 weeks) of sunitinib therapy in 23 patients. 16 (69.6\%) were classified as $\mathrm{RR}(\mathrm{PR}+\mathrm{SD})$ and the remaining 7 as $\mathrm{NR}$
(PD, 30.4\%). In the RR, median baseline RECIST and mRECIST derived tumor burden was $9.50 \mathrm{~cm}$ and $10.14 \mathrm{~cm}$ with a median percent change of $1.15 \%$ and $1.94 \%$, whereas in the NR the median tumor burden was $10.30 \mathrm{~cm}$ with a median percent change of $33.98 \%$ (baseline $\mathrm{P}=0.255$ and percent change $\mathrm{P}=0.0002$ ) for RECIST and $6.12 \mathrm{~cm}$ with a median percent change of $0.49 \%$ (baseline $\mathrm{P}=0.290$ and percent change $\mathrm{P}=$ 0.360). The median baseline tumor thrombus diameter in RR and NR was $26.42 \mathrm{~mm}$ and $31.48 \mathrm{~mm}(P=0.110)$ without any significant change at the end of sunitinib treatment $(-10.22 \%$ and $7.72 \%, P=0.260)$. Likewise, although the median baseline ADC value was relatively higher in NR, there was no significant difference in the median baseline ADC value as well as the median percent change of HCC and tumor thrombus between RR and NR.

In contrast, Ktrans in RR showed significantly larger median percent change $(-54.98 \%)$ than NR $(-17.83 \%)$ $(P=0.014)$. Similarly, Kep in RR showed significantly larger median percent change $(-42.06 \%)$ than NR $(-24.21 \%)(P=0.048)$. Moreover, the median percent Ktrans change of tumor thrombus in RR $(-55.00 \%)$ was 
Table 2 Correlation of DWI and MRP parameters with clinical outcome in HCC and tumor thrombus

\begin{tabular}{|c|c|c|c|c|c|c|c|}
\hline \multirow{2}{*}{ Parameters } & & \multicolumn{3}{|c|}{ Baseline } & \multicolumn{3}{|c|}{ Percent changes (\%) } \\
\hline & & $\begin{array}{l}\text { SD or PR } \\
\text { (Median, Range) }\end{array}$ & $\begin{array}{l}\text { PD } \\
\text { (Median, Range) }\end{array}$ & $P$ value & $\begin{array}{l}\text { SD or PR } \\
\text { (Median, Range) }\end{array}$ & $\begin{array}{l}\text { PD } \\
\text { (Median, Range) }\end{array}$ & $P$ value \\
\hline \multicolumn{8}{|l|}{ RECIST } \\
\hline \multicolumn{2}{|l|}{$H C C(\mathrm{~cm})$} & $9.50,2.30 \sim 17.00$ & $10.30,4.30 \sim 10.3$ & 0.255 & $1.15,-43.48 \sim 8.46$ & $33.98,20.71 \sim 58.23$ & 0.0002 \\
\hline \multicolumn{2}{|c|}{ Tumor thrombus $(\mathrm{mm})$} & $26.42,22.14 \sim 30.70$ & $31.48,24.65 \sim 38.34$ & 0.110 & $-10.22,-25.62 \sim 10.26$ & $7.72,-28.52 \sim 42.92$ & 0.260 \\
\hline \multicolumn{8}{|l|}{ mRECIST } \\
\hline \multicolumn{2}{|l|}{$H C C(\mathrm{~cm})$} & $10.14,2.15 \sim 19.56$ & $6.12,2.24 \sim 15.86$ & 0.290 & $1.94,-19.80 \sim 15.86$ & $0.49,-19.84 \sim 6.70$ & 0.360 \\
\hline \multicolumn{8}{|l|}{ DWI } \\
\hline $\mathrm{HCC}$ & $A D C\left(\times 10^{-3} \mathrm{~mm}^{2} / \mathrm{s}\right)$ & $0.86,0.58 \sim 0.99$ & $0.93,0.8 \sim 1.09$ & 0.15 & $22.42,-8.99 \sim 51.72$ & $22.22,-12.84 \sim 25.81$ & 0.300 \\
\hline Tumor thrombus & $A D C\left(\times 10^{-3} \mathrm{~mm}^{2} / \mathrm{s}\right)$ & $0.66,0.60 \sim 0.73$ & $0.96,0.79 \sim 1.14$ & 0.290 & $13.34,-13.28 \sim 39.94$ & $20.32,-62.33 \sim 114.23$ & 0.140 \\
\hline \multicolumn{8}{|l|}{ MRP } \\
\hline \multirow[t]{3}{*}{$H C C$} & Ktrans $\left(\min ^{-1}\right)$ & $2.35,0.90 \sim 4.81$ & $1.29,1.00 \sim 2.80$ & 0.402 & $-54.98,-83.37 \sim-10.00$ & $-17.83,-72.09 \sim-6.00$ & 0.014 \\
\hline & $\operatorname{Kep}\left(\min ^{-1}\right)$ & $4.25,1.00 \sim 41.75$ & $1.54,1.00 \sim 3.01$ & 0.18 & $-54.73,-96.32 \sim-5.04$ & $-12.19,-70.17 \sim 28.04$ & 0.048 \\
\hline & EVF & $0.83,0.34 \sim 1.00$ & $0.95,0.84 \sim 1.00$ & 0.065 & $-2.38,-47.50 \sim 61.76$ & $-6.45,-27.37 \sim-2.38$ & 0.331 \\
\hline \multirow[t]{3}{*}{ Tumor thrombus } & Ktrans $\left(\min ^{-1}\right)$ & $1.96,0.93 \sim 2.99$ & $1.73,1.05 \sim 2.41$ & 0.350 & $-55.00,-72.65 \sim-47.35$ & $-20.04,-63.23 \sim 24.14$ & 0.006 \\
\hline & $\operatorname{Kep}\left(\min ^{-1}\right)$ & $2.54,1.05 \sim 4.03$ & $1.97,1.18 \sim 2.76$ & 0.230 & $-45.53,-67.42 \sim-23.64$ & $-30.58,-80.01 \sim 18.98$ & 0.090 \\
\hline & $E V F$ & $0.81,0.70 \sim 0.92$ & $0.89,0.82 \sim 0.98$ & 0.110 & $-15.70,-31.95 \sim 0.56$ & $-29.65,-38.12 \sim-21.07$ & 0.060 \\
\hline
\end{tabular}

Abbreviations: Ktrans Transfer constant; Kep Rate constant; EVF Extracellular volume fraction; ADC Apparent diffusion coefficient; SD Partial disease; $P R$ Partial response; $P D$ Progressive disease.

also larger than that in NR $(-20.04 \%)(P=0.006)$. The median baseline as well as the median percent changes of EVF values in HCC and tumor thrombus didn't show any significant difference between $R R$ and NR (Table 2).
Correlation of baseline and percent changes in DWI and MRP parameters at 2-weeks with PFS

We then examined the correlation of baseline and percent changes in DWI and MRP parameters with PFS. The median PFS time of study cohort was 4.6 months

Table 3 Correlation of DWI and MRP parameters with PFS in HCC and tumor thrombus

\begin{tabular}{|c|c|c|c|c|c|c|c|}
\hline \multirow{2}{*}{ Parameters } & & \multicolumn{3}{|c|}{ Baseline } & \multicolumn{3}{|c|}{ Percent changes (\%) } \\
\hline & & $\begin{array}{l}\text { PFS }>6 \text { months } \\
\text { (Median, Range) }\end{array}$ & $\begin{array}{l}\text { PFS6 } \leq \text { months } \\
\text { (Median, Range) }\end{array}$ & $P$ value & $\begin{array}{l}\text { PFS }>6 \text { months } \\
\text { (Median, Range) }\end{array}$ & $\begin{array}{l}\text { PFS6 } \leq \text { months } \\
\text { (Median, Range) }\end{array}$ & $P$ value \\
\hline \multicolumn{8}{|l|}{ RECIST } \\
\hline \multicolumn{2}{|l|}{$H C C(\mathrm{~cm})$} & $10.00,5.20 \sim 14.00$ & $10.00,2.30 \sim 17.00$ & 0.682 & $3.57,-36.54 \sim 35.00$ & $7.60,-43.48 \sim 58.23$ & 0.875 \\
\hline \multicolumn{2}{|c|}{ Tumor thrombus(mm) } & $23.87,22.14 \sim 28.50$ & $30.79,24.65 \sim 38.34$ & 0.114 & $-15.23,-25.62 \sim 10.26$ & $9.72,-28.52 \sim 42.92$ & 0.019 \\
\hline \multicolumn{8}{|c|}{ mRECIST } \\
\hline \multicolumn{2}{|l|}{$H C C(\mathrm{~cm})$} & $10.70,2.15 \sim 19.56$ & $9.66,2.24 \sim 15.86$ & 0.207 & $-0.88,-11.16 \sim 11.00$ & $1.89,-19.84 \sim 15.86$ & 0.517 \\
\hline \multicolumn{8}{|l|}{ DWI } \\
\hline $\mathrm{HCC}$ & $A D C\left(\times 10^{-3} \mathrm{~mm}^{2} / \mathrm{s}\right)$ & $0.83,0.65 \sim 1.01$ & $0.90,0.69 \sim 1.09$ & 0.570 & $19.48,-12.84 \sim 40.00$ & $22.22,-8.99 \sim 51.72$ & 0.825 \\
\hline Tumor thrombus & $A D C\left(\times 10^{-3} \mathrm{~mm}^{2} / \mathrm{s}\right)$ & $0.74,0.60 \sim 1.03$ & $0.93,0.79 \sim 1.14$ & 0.085 & $10.38,-13.28 \sim 39.94$ & $21.11,-62.33 \sim 114.23$ & 0.315 \\
\hline \multicolumn{8}{|l|}{ MRP } \\
\hline \multirow[t]{3}{*}{$\mathrm{HCC}$} & Ktrans $\left(\min ^{-1}\right)$ & $2.80,1.00 \sim 4.81$ & $1.70,0.90 \sim 4.1$ & 0.043 & $-52.27,-83.37 \sim-7.00$ & $-41.25,-75.61 \sim-6.00$ & 0.467 \\
\hline & Kep $\left(\min ^{-1}\right)$ & $3.01,1.00 \sim 41.75$ & $2.79,1.27 \sim 4.36$ & 0.100 & $-49.58,-96.32 \sim 28.04$ & $-46.33,-77.05 \sim-1.67$ & 0.914 \\
\hline & EVF & $0.93,0.57 \sim 1.00$ & $0.88,0.34 \sim 1.00$ & 0.590 & $-8.77,-47.50 \sim 20.55$ & $-2.38,-30.12 \sim 61.76$ & 0.022 \\
\hline \multirow[t]{3}{*}{ Tumor thrombus } & Ktrans $\left(\min ^{-1}\right)$ & $1.65,0.93 \sim 2.33$ & $1.98,1.05 \sim 2.99$ & 0.374 & $-58.48,-72.65 \sim-47.35$ & $-23.11,-63.23 \sim 24.14$ & 0.005 \\
\hline & $\operatorname{Kep}\left(\min ^{-1}\right)$ & $2.43,1.32 \sim 4.03$ & $1.95,1.05 \sim 2.76$ & 0.220 & $-42.53,-67.42 \sim-23.64$ & $-34.58,-80.01 \sim 18.98$ & 0.135 \\
\hline & EVF & $0.78,0.70 \sim 0.92$ & $0.88,0.82 \sim 0.98$ & 0.120 & $-11.30,-31.95 \sim 0.56$ & $-22.43,-38.12 \sim-19.75$ & 0.331 \\
\hline
\end{tabular}

Abbreviations: Ktrans Transfer constant; Kep Rate constant; EVF Extracellular volume fraction; ADC Apparent diffusion coefficient. 
(95\% confidence interval [CI], $3.8 \sim 8.9$ months) and the updated overall survival time was 9.9 months $(95 \%$ CI, $7.5 \sim 14.1$ months). The PFS rate at 6 months was $39.1 \%$.

The median baseline ADC value was relatively higher in patients with PFS $\leq 6$ months but not statistically different from the median baseline ADC value as well as the median percent change of HCC and tumor thrombus between these two groups.

In contrast, the median baseline Ktrans in patients with PFS $>6$ months $\left(2.80 \mathrm{~min}^{-1}\right)$ was significantly higher than that in those with PFS $\leq 6$ months $\left(1.70 \mathrm{~min}^{-1}\right)$ $(P=0.043)$. Moreover, the median percent change of tumor thrombus in patients with PFS $>6$ months $(-58.48 \%)$ was also larger than those with PFS $\leq 6$ months $(-23.11 \%)$ $(P=0.005)$. The median percent changes of EVF value in

\section{Table 4 Correlation between changes in MRI parameters and plasma biomarkers after sunitinib treatment (Kendall's $\tau$ correlation coefficients)}

\begin{tabular}{|c|c|c|c|c|}
\hline Kendall's T & Ktrans & Kep & $E V F$ & $A D C$ \\
\hline VEGF & $0.00^{*}$ & 0.11 & 0.03 & 0.24 \\
\hline$P$-value & 1.00 & 0.49 & 0.86 & 0.13 \\
\hline PIGF & 0.00 & 0.10 & -0.06 & 0.06 \\
\hline$P$-value & 0.74 & 0.53 & 0.72 & 0.72 \\
\hline sVEGFR1 & 0.22 & 0.18 & -0.16 & -0.16 \\
\hline$P$-value & 0.16 & 0.26 & 0.30 & 0.30 \\
\hline sVEGFR2 & 0.33 & 0.35 & -0.05 & -0.19 \\
\hline$P$-value & 0.044 & 0.030 & 0.77 & 0.24 \\
\hline sVEGFR3 & 0.00 & -0.09 & 0.24 & -0.23 \\
\hline$P$-value & 1.00 & 0.62 & 0.21 & 0.22 \\
\hline VEGF-C & 0.17 & 0.17 & 0.02 & -0.01 \\
\hline$P$-value & 0.28 & 0.29 & 0.90 & 0.95 \\
\hline bFGF & 0.17 & 0.15 & 0.01 & -0.06 \\
\hline$P$-value & 0.28 & 0.45 & 0.86 & 0.72 \\
\hline TNF- $a$ & 0.32 & 0.34 & 0.01 & -0.17 \\
\hline$P$-value & 0.051 & 0.030 & 0.97 & 0.30 \\
\hline SDF1a & 0.02 & 0.14 & 0.12 & 0.26 \\
\hline$P$-value & 0.90 & 0.38 & 0.47 & 0.10 \\
\hline IL-1 $\beta$ & 0.16 & 0.14 & 0.16 & -0.02 \\
\hline$P$-value & 0.33 & 0.38 & 0.31 & 0.90 \\
\hline IL-6 & 0.01 & -0.04 & -0.04 & -0.13 \\
\hline$P$-value & 0.95 & 0.82 & 0.82 & 0.44 \\
\hline IL-8 & 0.10 & 0.08 & 0.04 & -0.21 \\
\hline$P$-value & 0.56 & 0.63 & 0.82 & 0.19 \\
\hline Sol c-KIT & 0.20 & 0.12 & -0.01 & 0.01 \\
\hline$P$-value & 0.20 & 0.45 & 0.95 & 0.95 \\
\hline
\end{tabular}

*Positive values of Kendall's $\tau$ indicate a direct correlation between changes after treatment (relative to baseline) in imaging biomarkers and plasma biomarkers.
HCC showed a significant correlation with PFS $(P=0.022)$ (Table 3).

\section{Changes in vascular permeability measured by MRI and circulating biomarkers}

The changes in circulating biomarkers at 2 weeks after sunitinib treatment have been reported elsewhere [10]. When we compared the change in MRI parameters with the change in plasma angiogenic and inflammatory cytokines we found a significant correlation between decrease in sVEGFR2 or TNF- $\alpha$ and a drop in Kep $(P=0.030$ and $P=0.035$ respectively) and a similar correlation for the decrease in Ktrans $(P=0.044$ and $P=0.051$ respectively). There was no other association between the changes in MRI parameters and circulating biomarkers at this timepoint (Table 4).

\section{Discussion}

The RECIST based change in tumor burden following treatment with chemotherapy is a widely accepted imaging surrogate for assessing treatment outcome in oncologic clinical trials. Its ease of use, quantization and reproducibility has been the major attribute for its success. Due to deficiencies in RECIST for evaluating treatment efficacy in HCC, the criteria have been modified (mRECIST) to include arterial phase enhancement of the lesion [16]. However, novel targeted antiangiogenic approaches may induce necrosis and stabilize tumor growth rather than tumor regression, which makes the early response evaluation challenging. In this context, there has been an increase in the utilization of MRP in $\mathrm{HCC}$, including for monitoring early therapeutic effects after a few days/weeks of antiangiogenic treatment $[12,26,27]$. An advantage of the MRP technique is that it can be incorporated into routine conventional MRI providing physiological information. Moreover, MRP coupled with powerful and user-friendly software packages can offer excellent contrast resolution, more coverage, repeated examinations and continuous sampling of data for more than four minutes, which allows the assessment of washout without exposure to ionizing radiation.

In this study, we observed that the MRP derived HCC parameters (Ktrans and Kep) were more sensitive imaging biomarkers than ADC value, RECIST, and mRECIST for monitoring early antiangiogenic treatment effects. Ktrans (wash-in rate) describes the leakage rate of the contrast medium. For blood vessels where leakage is rapid, that is, when extraction fraction during the first pass of the contrast agent is high, perfusion will determine contrast agent distribution and Ktrans approximates to tissue blood flow per unit volume [25]. Whereas, Kep measures the rate of contrast agent diffusion back into the vasculature (washout rate) from where it is excreted [25]. Higher baseline 
Ktrans value and more substantial drop in Ktrans and Kep at 2 weeks after therapy correlated with better clinical outcome or PFS. Our results support the hypothesis that after antiangiogenic therapy, the changes in tumor perfusion precede the change in tumor size, which make the MRP parameters more sensitive for monitoring early antiangiogenic effects compared with tumor burden measurements as defined by RECIST or mRECIST in advanced HCC. The reduced tumor vessel permeability as estimated by MRP indicated a direct effect on HCC microvasculature that might be associated with clinical benefit after sunitinib therapy. Similar observations have been reported by de Langen $\mathrm{AJ}$ et al. in patients with advanced non-small cell lung cancer treated with bevacizumab and erlotinib [28]. In advanced HCC, DCE-MRI demonstrated reduction in Ktrans during antiangiogenic treatment and the change of Ktrans during treatment was related to better PFS and OS in clinical trials of sorafenib [26,27]. The change of Ktrans may reflect the underlying tumor permeability changes induced by antiangiogenic therapy. This suggests that control of vessel leakiness may be a determinant of HCC response to sunitinib $[28,29]$.

In addition, we found the higher baseline of Ktrans can even relate with longer PFS. Similar studies on the predictive value of tumor perfusion parameters have also been reported. In cervical cancer, the MRP parameters quantifying permeability status can provide very early prediction of tumor control and disease-free-survival [30]. The MRP parameters such as Ktrans depend on vascular permeability and are being considered as imaging biomarker because they can detect functional changes in tumor vasculature after treatment with anti-VEGF agents [31]. The increased concentration gradient across the endothelial membrane, the larger surface area of the vascular endothelium to which they are exposed, the higher endothelial permeability, the loss of cell membrane integrity and higher cellular density can all contribute to the relatively higher baseline permeability values in responders and patients with longer PFS $[25,32,33]$.

Increase in $\mathrm{ADC}$ in response to sunitinib therapy is consistent with the observations made by other investigators on ADC increase in liver tumors to other nonsurgical treatments and is believed to result from loss of cell membrane integrity or necrosis [34,35]. However, the median baseline value and the percent change of ADC did not correlate with the clinical outcome and PFS. It is possibly due to higher biologic aggressiveness and presence of tumor necrosis. Poor perfusion is known to impede drug delivery and induce hypoxic and acidic environment, which diminishes the effectiveness of antiangiogenic therapy [36,37]. In addition, following antiangiogenic therapy, improvement of tumor perfusion, edema and inflammation may be the dominant factor to influence the ADC values instead of necrosis and loss of cell membrane integrity that often follows much later in the course of system chemotherapy [38]. This potentially explains mild changes in ADC in comparison to more significant changes in Ktrans and Kep.

Interestingly, we also found that tumor thrombus showed high baseline values and substantial reduction in perfusion parameters following sunitinib treatment almost similar to the response in the primary tumor. The accurate differentiation of bland from tumor thrombus is crucial for patient treatment. Although a neoplastic thrombus can be discriminated from a clot in most cases by CE-MRI alone, characterization of small thrombi or a peripheral one can be difficult on conventional MR alone. Our results support the tissue characterization benefits of MRP parameters as well as DWI, which could potentially be applied in differentiating tumor thrombus from bland thrombus [23]. Moreover, tumor thrombus could be the only visible evidence of measurable disease and might be used for response evaluation.

Signaling through VEGFR2 in endothelial cells is critical in VEGF-induced vascular leakiness [39,40], and a decrease in circulating sVEGFR2 has been consistently seen with all agents that block this pathway $[10,41,42]$. Similarly, TNF- $\alpha$-a pro-inflammatory cytokine-is known to increase vascular permeability [43]. The preliminary finding that the changes in MRI parameters are associated with the changes in circulating sVEGFR2 and TNF- $\alpha$ suggests that the rapid drop in vessel leakiness in HCC after sunitinib treatment may potentially occur by direct blockade of VEGF/VEGFR2 signaling or indirectly by reduction of TNF- $\alpha$. In this study, Kep showed significant correlations with both VEGFR2 and TNF- $\alpha$, whereas Ktrans showed a significant correlation with only VEGFR2. There was a trend for correlation between Ktrans and TNF- $\alpha$, but it did not reach statistical significance, which may be due to the small sample size of this study. These associations should be tested in larger prospective studies.

It should be noted that our study has a few limitations. First, the sample size is relatively small and the predictive value of Ktrans and Kep remains to be validated in larger prospective studies. In addition, the diagnosis of tumor invasion in the portal vein was based on wellestablished imaging criteria of portal vein expansion and appreciable enhancement in the thrombus and not on histopathologic sampling.

\section{Conclusions}

Our experience from this phase II study suggests that following antiangiogenic therapy in advanced HCC, imaging changes are detectable within 2 weeks on DWI and MRP-derived parameters. Moreover, larger percent drops in Ktrans and EFV - but not the changes in tumor burden - correlated with longer PFS, which suggests that 
they are potentially superior imaging biomarkers of response for antiangiogenic therapy in HCC. These results may be specific to the method of analysis and the software employed in this study and warrant validation in future studies. However, these data indicate that MRI-based evaluations of tumor diffusion and perfusion and circulating biomarker evaluation will not only provide a better mechanistic understanding of the effects of antiangiogenic therapies, but will also facilitate tumor response assessment.

\section{Abbreviations \\ ADC: Apparent diffusion coefficient; DWI: Diffusion-weighted imaging; EVF: Extracellular volume fraction; HCC: Hepatocellular carcinoma; Ktrans: Transfer constant; Kep: Redistribution rate constant; MRI: Magnetic resonance imaging; MRP: MR perfusion; PFS: Progression free survival; RECIST: Response Evaluation, Criteria In Solid Tumors; ROI: Region of interest; VEGF: Vascular endothelial growth factor.}

\section{Competing interests}

Andrew X Zhu, M.D. served as a consultant for Pfizer. Rakesh K. Jain received research grants from Dyax, Medlmmune and Roche; received consultant fees from Dyax, Enlight, Noxxon, and Zyngenia; owns equity in Enlight, SynDevRx, and XTuit; and serves on the Board of Directors of XTuit and Boards of Trustees of H\&Q Healthcare Investors and H\&Q Life Sciences Investors. No reagents or funds from these organizations were used in this study. All other authors declare no conflicts.

\section{Authors' contributions}

DVS participated in data collection, data analysis, prepared the manuscript for submission and manuscript preparation. TJ participated in data collection, data analysis and prepared the manuscript for submission. $\mathrm{KH}$ prepared the manuscript for submission, participated in data collection, data analysis and manuscript revision. DGD participated in data collection, data analysis and manuscript preparation. OAC participated in data collection, data analysis and manuscript preparation. AM participated in data collection, data analysis and manuscript revision. RKJ participated in treatment planning, data collection, data analysis and manuscript preparation. AXZ participated in treatment planning and manuscript preparation. All authors have read and approved the final manuscript.

\section{Acknowledgments}

Tao Jiang, M.D. received research support from the National Natural Science Foundation of China (No.81101044). The research of Drs. Jain and Duda is in part supported through $\mathrm{NIH}$ grant P01-CA080124.

\section{Author details}

'Division of Abdominal Imaging and Intervention, Harvard Medical School and Massachusetts General Hospital, 55 Fruit Street, White 270, Boston, MA 02114, USA. ${ }^{2}$ Department of Radiology, ChangZheng Hospital Affiliated to Second Military Medical University, 415 Fengyang Road, Shanghai 200003, China. ${ }^{3}$ Steele Laboratory, Massachusetts General Hospital, 100 Blossom Street, Cox-734, Boston, MA 02114, USA. ${ }^{4}$ Department of Radiology, SDN Foundation, IRCCS, 80143, Naples, Italy. ${ }^{5}$ Cancer Center, Massachusetts General Hospital, 55 Fruit Street, LH/POB 232, Boston, MA 02114, USA.

Received: 24 May 2013 Accepted: 26 June 2013

Published: 10 July 2013

\section{References}

1. Jemal A, Siegel R, Xu J, Ward E: Cancer statistics, 2010. CA Cancer J Clin 2010, 60:277-300.

2. Giglia JL, Antonia SJ, Berk LB, Bruno S, Dessureault S, Finkelstein SE: Systemic therapy for advanced hepatocellular carcinoma: past, present, and future. Cancer Control 2010, 17:120-129.

3. Thomas MB: Systemic therapy for hepatocellular carcinoma. Cancer J 2008, 14:123-127.

4. Murukesh N, Dive C, Jayson GC: Biomarkers of angiogenesis and their role in the development of VEGF inhibitors. Br J Cancer 2010, 102:8-18.
5. Wörns MA, Schuchmann M, Düber C, Otto G, Galle PR, Weinmann A: Sunitinib in patients with advanced hepatocellular carcinoma after progression under sorafenib treatment. Oncology 2010, 79:85-92.

6. Thomas MB, Chadha R, Glover K, Wang X, Morris J, Brown T, Rashid A, Dancey J, Abbruzzese JL: Phase 2 study of erlotinib in patients with unresectable hepatocellular carcinoma. Cancer 2007, 110:1059-1067.

7. Siegel AB, Cohen El, Ocean A, Lehrer D, Goldenberg A, Knox JJ, Chen H, Clark-Garvey S, Weinberg A, Mandeli J, Christos P, Mazumdar M, Popa E, Brown RS Jr, Rafii S, Schwartz JD: Phase II trial evaluating the clinical and biologic effects of bevacizumab in unresectable hepatocellular carcinoma. J Clin Oncol 2008, 26:2992-2998.

8. Zhu AX, Duda DG, Sahani DV, Jain RK: Development of sunitinib in hepatocellular carcinoma: rationale, early clinical experience, and correlative studies. Cancer J 2009, 15:263-268.

9. Zhu AX, Holalkere NS, Muzikansky A, Horgan K, Sahani DV: Early antiangiogenic activity of bevacizumab evaluated by computed tomography perfusion scan in patients with advanced hepatocellular carcinoma. Oncologist 2008, 13:120-125.

10. Zhu AX, Ancukiewicz M, Supko JG, Sahani DV, Blaszkowsky LS, Meyerhardt JA Abrams TA, McCleary NJ, Bhargava P, Muzikansky A, Sheehan S, Regan E, Vasudev E, Knowles M, Fuchs CS, Ryan DP, Jain RK, Duda DG: Efficacy, safety, pharmacokinetics, and biomarkers of cediranib monotherapy in advanced hepatocellular carcinoma: a phase II study. Clin Cancer Res 2013, 19:1557-1566

11. Zhu AX, Duda DG, Sahani DV, Jain RK: HCC and angiogenesis: possible targets and future directions. Nat Rev Clin Oncol 2011, 8:292-301.

12. Zhu AX, Sahani DV, Duda DG, di Tomaso E, Ancukiewicz M, Catalano OA, Sindhwani V, Blaszkowsky LS, Yoon SS, Lahdenranta J, Bhargava P, Meyerhardt J, Clark JW, Kwak EL, Hezel AF, Miksad R, Abrams TA, Enzinger PC, Fuchs CS, Ryan DP, Jain RK: Efficacy, safety, and potential biomarkers of sunitinib monotherapy in advanced hepatocellular carcinoma: a phase II study. J Clin Oncol 2009, 27:3027-3035.

13. Faivre S, Raymond E, Boucher E, Douillard J, Lim HY, Kim JS, Zappa M, Lanzalone S, Lin X, Deprimo S, Harmon C, Ruiz-Garcia A, Lechuga MJ, Cheng AL: Safety and efficacy of sunitinib in patients with advanced hepatocellular carcinoma: an open-label, multicentre, phase II study. Lancet Oncol 2009, 10:794-800.

14. Marcus CD, Ladam-Marcus V, Cucu C, Bouche O, Lucas L, Hoeffel C: Imaging techniques to evaluate the response to treatment in oncology: current standards and perspectives. Crit Rev Oncol Hematol 2009, 72:217-238.

15. Bruix J, Sherman M, Llovet JM, Beaugrand M, Lencioni R, Burroughs AK, Christensen E, Pagliaro L, Colombo M, Rodés J: EASL panel of experts on HCC: clinical management of hepatocellular carcinoma. Conclusions of the barcelona-2000 EASL conference. European association for the study of the liver. J Hepatol 2001, 35:421-430.

16. Llovet JM, Di Bisceglie AM, Bruix J, Kramer BS, Lencioni R, Zhu AX, Sherman M, Schwartz M, Lotze M, Talwalkar J, Gores GJ: Panel of experts in HCC-design clinical trials: design and endpoints of clinical trials in hepatocellular carcinoma. J Natl Cancer Inst 2008, 100:698-711.

17. Jiang T, Zhu AX, Sahani DV: Established and novel imaging biomarkers for assessing response to therapy in hepatocellular carcinoma. J Hepatol 2013, 58:169-177.

18. Zech CJ, Reiser MF, Herrmann KA: Imaging of hepatocellular carcinoma by computed tomography and magnetic resonance imaging: state of the art. Dig Dis 2009, 27:114-124.

19. Jarnagin WR, Schwartz LH, Gultekin DH, Gönen M, Haviland D, Shia J, D'Angelica M, Fong Y, Dematteo R, Tse A, Blumgart LH, Kemeny N: Regional chemotherapy for unresectable primary liver cancer: results of a phase II clinical trial and assessment of DCE-MRI as a biomarker of survival. Ann Oncol 2009, 20:1589-1595.

20. Kubota K, Yamanishi T, Itoh S, Murata Y, Miyatake K, Yasunami H, Morio K, Hamada N, Nishioka A, Ogawa Y: Role of diffusion-weighted imaging in evaluating therapeutic efficacy after transcatheter arterial chemoembolization for hepatocellular carcinoma. Oncol Rep 2010, 24:727-732.

21. Chung JC, Naik NK, Lewandowski RJ, Deng J, Mulcahy MF, Kulik LM, Sato KT, Ryu RK, Salem R, Larson AC, Omary RA: Diffusion-weighted magnetic resonance imaging to predict response of hepatocellular carcinoma to chemoembolization. World J Gastroenterol 2010, 16:3161-3167.

22. Shah ZK, McKernan MG, Hahn PF, Sahani DV: Enhancing and expansile portal vein thrombosis: value in the diagnosis of hepatocellular 
carcinoma in patients with multiple hepatic lesions. AJR Am J Roentgenol 2007, 188:1320-1323.

23. Catalano OA, Choy G, Zhu A, Hahn PF, Sahani DV: Differentiation of malignant thrombus from bland thrombus of the portal vein in patients with hepatocellular carcinoma: application of diffusion-weighted MR imaging. Radiology 2010, 254:154-162.

24. Yang C, Karczmar GS, Medved M, Stadler WM: Estimating the arterial input function using two reference tissues in dynamic contrast-enhanced MRI studies: fundamental concepts and simulations. Magn Reson Med 2004, 52:1110-1117.

25. Tofts PS, Brix G, Buckley DL, Evelhoch JL, Henderson E, Knopp MV, Larsson HB, Lee TY, Mayr NA, Parker GJ, Port RE, Taylor J, Weisskoff RM: Estimating kinetic parameters from dynamic contrast-enhanced T(1)-weighted MRI of a diffusable tracer: standardized quantities and symbols. J Magn Reson Imaging 1999, 10:223-232.

26. Yau T, Chen PJ, Chan P, Curtis CM, Murphy PS, Suttle AB, Gauvin J, Hodge JP, Dar MM, Poon RT: Phase I dose-finding study of pazooanib in hepatocellular carcinoma: evaluation of early efficacy, pharmacokinetics, and pharmacodynamics. Clin Cancer Res 2011, 17:6914-6923.

27. Hsu CY, Shen YC, Yu CW, Hsu C, Hu FC, Hsu CH, Chen BB, Wei SY, Cheng AL, Shih TTF: Dynamic contrst-enhanced magnetic resonance imaging biomarkers predict survival and response in hepatocellular carcinoma patients treated with sorafenib and metronomic tegafur/uracil. $J$ Hepatol 2011, 55:858-865.

28. de Langen AJ, van den Boogaart $\mathrm{V}$, Lubberink M, Backes WH, Marcus JT, van Tinteren $H$, Pruim J, Brans B, Leffers $P$, Dingemans AM, Smit EF, Groen HJ, Hoekstra OS: Monitoring response to antiangiogenic therapy in non-small cell lung cancer using imaging markers derived from PET and dynamic contrast-enhanced MRI. J Nucl Med 2011, 52:48-55.

29. Pickles MD, Lowry M, Manton DJ, Gibbs P, Turnbull LW: Role of dynamic contrast enhanced MRI in monitoring early response of locally advanced breast cancer to neoadjuvant chemotherapy. Breast Cancer Res Treat 2005, 91:1-10.

30. Yuh WT, Mayr NA, Jarjoura D, Wu D, Grecula JC, Lo SS, Edwards SM, Magnotta VA, Sammet S, Zhang H, Montebello JF, Fowler J, Knopp MV Wang JZ: Predicting control of primary tumor and survival by DCE MRI during early therapy in cervical cancer. Invest Radiol 2009, 44:343-350.

31. Morgan B, Thomas AL, Drevs J, Hennig J, Buchert M, Jivan A, Horsfield MA Mross K, Ball HA, Lee L, Mietlowski W, Fuxuis S, Unger C, O'Byrne K, Henry A, Cherryman GR, Laurent D, Dugan M, Marmé D, Steward WP: Dynamic contrast-enhanced magnetic resonance imaging as a biomarker for the pharmacological response of PTK787/ZK 222584, an inhibitor of the vascular endothelial growth factor receptor tyrosine kinases, in patients with advanced colorectal cancer and liver metastases: results from two phase I studies. J Clin Oncol 2003, 21:3955-3964.

32. Haroon HA, Buckley DL, Patankar TA, Dow GR, Rutherford SA, Balériaux D, Jackson A: A comparison of ktrans measurements obtained with conventional and first pass pharmacokinetic models in human gliomas. J Magn Reson Imaging 2004, 19:527-536.

33. Barboriak DP, MacFall JR, Viglianti BL, Dewhirst-Dvm MW: Comparison of three physiologically-based pharmacokinetic models for the prediction of contrast agent distribution measured by dynamic MR imaging. J Magn Reson Imaging 2008, 27:1388-1398.

34. Koh DM, Scurr E, Collins D, Kanber B, Norman A, Leach MO, Husband JE: Predicting response of colorectal hepatic metastasis: value of pretreatment apparent diffusion coefficients. AJR Am J Roentgenol 2007, 188:1001-1008.

35. Kamel IR, Bluemke DA, Eng J, Liapi E, Messersmith W, Reyes DK, Geschwind JF: The role of functional MR imaging in the assessment of tumor response after chemoembolization in patients with hepatocellular carcinoma. J Vasc Interv Radiol 2006, 17:505-512.

36. Harrison L, Blackwell K: Hypoxia and anemia: factors in decreased sensitivity to radiation therapy and chemotherapy? Oncologist 2004, 9(Suppl 5):31-40.

37. Jain RK: Normalizing tumor microenvironment to treat cancer: bench to bedside to biomarkers. J Clin Oncol 2013, 31:2205-2218.

38. Hamstra DA, Rehemtulla A, Ross BD: Diffusion magnetic resonance imaging: a biomarker for treatment response in oncology. $J$ Clin Oncol 2007, 25:4104-4109.
39. Dvorak HF: Vascular permeability factor/vascular endothelial growth factor: a critical cytokine in tumor angiogenesis and a potential target for diagnosis and therapy. J Clin Oncol 2002, 20:4368-4380.

40. Carmeliet $P$, Jain RK: Molecular mechanisms and clinical applications of angiogenesis. Nature 2011, 473:298-307.

41. Batchelor TT, Sorensen AG, di Tomaso E, Zhang WT, Duda DG, Cohen KS, Kozak KR, Cahill DP, Chen PJ, Zhu M, Ancukiewicz M, Mrugala MM, Plotkin S, Drappatz J, Louis DN, Ivy P, Scadden DT, Benner T, Loeffler JS, Wen PY, Jain RK: AZD2171, A pan-VEGF receptor tyrosine kinase inhibitor, normalizes tumor vasculature and alleviates edema in glioblastoma patients. Cancer Cell 2007, 11:83-95.

42. Motzer RJ, Michaelson MD, Redman BG, Hudes GR, Wilding G, Figlin RA, Ginsberg MS, Kim ST, Baum CM, DePrimo SE, Li JZ, Bello CL, Theuer CP, George DJ, Rini BI: Activity of SU11248, a multitargeted inhibitor of vascular endothelial growth factor receptor and platelet-derived growth factor receptor, in patients with metastatic renal cell carcinoma. J Clin Oncol 2006, 24:16-24.

43. Lejeune FJ, Ruegg C: Recombinant human tumor necrosis factor: an efficient agent for cancer treatment. Bull Cancer 2006, 93:E90-E100.

doi:10.1186/1756-8722-6-51

Cite this article as: Sahani et al:: Magnetic resonance imaging biomarkers in hepatocellular carcinoma: association with response and circulating biomarkers after sunitinib therapy. Journal of Hematology \& Oncology 2013 6:51.

\section{Submit your next manuscript to BioMed Central and take full advantage of:}

- Convenient online submission

- Thorough peer review

- No space constraints or color figure charges

- Immediate publication on acceptance

- Inclusion in PubMed, CAS, Scopus and Google Scholar

- Research which is freely available for redistribution 